\title{
The hazards of conducting induction of labour in high-risk pregnancies at district hospitals in low- and middle-income countries: lessons to learn from a case report
}

\author{
Nnabuike Chibuoke Ngene ${ }^{1,2}$, Jagidesa Moodley ${ }^{3}$
}

1. Department of Obstetrics and Gynaecology, Klerksdorp Hospital, Klerksdorp, South Africa;

2. Department of Obstetrics and Gynaecology, School of Clinical Medicine, Faculty of Health Sciences, University of the Witwatersrand, Johannesburg, South Africa.

3. Women's Health and HIV Research Group, Department of Obstetrics and Gynaecology, Nelson R Mandela School of Clinical Medicine, University of KwaZulu-Natal, South Africa.

\section{Co-author details:}

Email: jmog@ukzn.ac.za

\begin{abstract}
Background: Induction of labour (IOL) is an obstetric procedure that should be conducted in a healthcare facility with the capacity to provide optimal care based on the patient risk status. Inadequate monitoring, untimely procedure and lack of readily available and experienced medical staff to participate in the care of the patient undergoing induction are hazardous with snowball effects.

Methods: A 38-year-old G4P2+1 had IOL because of oligohydramnios at term in a district hospital. The procedure was inadequately monitored and fetal demise occurred. The duration of second stage was prolonged and sequential use of vacuum and forceps deliveries were unsuccessfully performed.

Results: At the ensuing caesarean delivery, uterine rupture/tear was diagnosed, and the patient died due to haemorrhage during an emergency hysterectomy.

Conclusion: This report highlights important clinical lessons on IOL in a high-risk pregnancy. The timelines for monitoring during IOL, particularly when there is fetal demise in labour, are proposed.

Keywords: Assisted vaginal delivery, induction of labour, prolonged labour, oligohydramnios, uterine tear.

DOI: https://dx.doi.org/10.4314/ahs.v20i3.27

Cite as: Ngene NC, Moodley J. The hazards of conducting induction of labour in high-risk pregnancies at district hospitals in low- and middle-income countries: lessons to learn from a case report. Afri Health Sci. 2020;20(3): 1237-1240. https:// dx.doi. org/10.4314/ahs.v20i3.27
\end{abstract}

\section{Introduction}

Induction of labour (IOL) is an artificial commencement of labour ${ }^{1}$ to achieve vaginal delivery ${ }^{2}$. The process can lead to complications such as placental insuf-

\section{Corresponding author: \\ Nnabuike Chibuoke Ngene, \\ Department of Obstetrics and Gynaecology, \\ Klerksdorp Hospital, Klerksdorp, South Africa; \\ and \\ Department of Obstetrics and Gynaecology, \\ School of Clinical Medicine, Faculty of Health \\ Sciences, University of the Witwatersrand, \\ Johannesburg, South Africa. \\ P. O. Box 6873, Flamwood, 2572, \\ Klerksdorp, South Africa. \\ Email: ngenenc@gmail.com}

ficiency and uterine rupture which may be fatal. To prevent poor maternal and or fetal outcomes, care must be taken to ensure close monitoring of maternal conditions, safe labour practices including judicious oxytocic therapy, indicated operative vaginal delivery and timely caesarean delivery (CD). Unsafe practices such as the inability to monitor or detect prolonged labour are associated with harm ${ }^{3}$ particularly in a multigravida with previous vaginal birth (a patient group less likely to develop uterine inertia once in an active phase of labour). To prevent the complications associated with IOL, the procedure should be conducted in a healthcare facility with a full complement of resources including readily available and experienced medical staff capable of managing the patient's risk. Therefore, IOL on a high-risk obstetric patient should be conducted in a regional hospital or at a higher level of care. In the case presented, a 
high-risk obstetric patient had IOL in a district hospital $(\mathrm{DH})$ and this resulted in inadequate monitoring, fetal demise, prolonged second stage of labour, sequential use of vacuum and forceps deliveries, uterine rupture/ tear, CD, hysterectomy and maternal death. This case report is aimed to demonstrate important clinical lessons on the dangers of IOL in a high-risk pregnancy at a DH that lacks the capacity to adequately manage risks.

\section{Case presentation}

A 38-year-old gravida 4 para 2 with one previous miscarriage had her index antenatal care conducted in a district hospital. Her basic antenatal blood tests including haemoglobin concentration were normal. At 37 gestational weeks, ultrasonography to assess fetal wellbeing showed alive normal fetus with oligohydramnios (although the severity and method used for the assessment of the liquor volume were not recorded), and the patient was admitted for IOL.

The IOL was commenced in the morning with a normal pre-induction non-stress cardiotocography (fetal heart rate of $135 /$ minute). The pre-induction haemoglobin concentration and Bishop score were not assessed and recorded. Misoprostol $200 \mathrm{mcg}$ was dissolved in $200 \mathrm{ml}$ of drinking water and prescribed to be administered to the patient orally 2 hourly at a dose of $20 / 20 / 20 / 20 / 40 / 40 \mathrm{ml}$. Eight hours following commencement of IOL, the last dose of misoprostol given to the patient was administered and a medical officer noted that there were mild uterine contractions although the cervical dilatation was not assessed. Thereafter, there was no documentation of uterine contractions, the fetal heart rate, descent of the fetal head or assessment of cervical dilatation until 2.5 hours later when intrauterine fetal death was diagnosed at full cervical dilatation; the patient was draining clear liquor with a normal blood pressure, pulse, and temperature. After approximately 5 hours in the second stage of labour and 3 attempts at operative vaginal delivery (failed vacuum twice and forceps once) the patient was booked for CD. On that day, the hospital telephones were faulty preventing timely communication between the medical staff on duty. Pre-operative haemoglobin concentration was not performed. The surgical operation for the $\mathrm{CD}$ was commenced after approximately 8 hours in the second stage of labour. At CD, haemoperitoneum and uterine ruptures/tears were detected. There was a right posterior tear from the fundus to the cervix and another lateral tear on the left uterine body. A grossly normal fresh stillbirth weighing $2.8 \mathrm{~kg}$ was extracted. Repair of the uterine tears was commenced although difficult. While the tear was being repaired, the patient became hemodynamically unstable and the spinal anaesthesia was converted to general anaesthesia, senior help was invited, and a hysterectomy was performed. At the completion of the hysterectomy, the patient developed ventricular fibrillation and collapsed. All attempts at resuscitation including blood transfusion failed, and the patient was declared dead. The haemoglobin concentration during the resuscitation was $4.8 \mathrm{~g} / \mathrm{dl}$ while the total blood loss during the surgical procedure was $3 \mathrm{~L}$.

\section{Discussion}

Most DH in low- and middle-income countries particularly those in deep rural areas are poorly staffed by inexperienced medical officers. In addition, there is frequently a shortage of nursing staff and doctors at night. Therefore, triaging of "at risk patients" is critical in the practice of good clinical care. The patient was 38 years old and found to have oligohydramnios. It is well known that women over the age of 35 years have a higher risk of maternal morbidity and mortality ${ }^{4}$ and stillbirths ${ }^{2}$ than a younger age group. Therefore, the patient should have been triaged to a regional hospital which is better staffed, so that observations of the mother and fetus can be performed at frequent intervals according to a partogram. This case illustrates poor monitoring, poor recordings and poor use of the partogram in the first and second stages of labour and a failure to recognize the dangers of a prolonged second stage of labour.

Other lessons to learn from this case report are: (a) Maternal haemoglobin concentration should be assessed before an IOL. (b) Maternal and fetal conditions should be adequately monitored during an IOL. The frequency of monitoring has not been fully investigated ${ }^{1}$ but should be guided by the method of induction, feto-maternal condition and institutional protocol. Fetal heart monitoring, preferably electronic, should be performed before commencing IOL and within one hour after each time prostaglandin or its analogue is administered ${ }^{5}$. If vaginal prostaglandin $\mathrm{E}^{2}$ tablet or gel is used, an assessment should be conducted not later than 6 hours following the therapy ${ }^{1}$. Vaginal examination may be arguably performed using the time limits set for latent phase of labour in the maternity unit or conducted earlier if there are uterine contractions or any other indication, but the examination should be repeated not later 
than every 6 hours. (c) In a term pregnancy, prolonged duration of labour may be hazardous to a mother and her baby. The second stage of labour should not exceed 3 hours in a primigravida and 2 hours in a multigravida ${ }^{6}$. A study $(\mathrm{n}=78)$ showing that extended time limits for labour in primigravidae (with singleton fetuses in cephalic presentation) reduces $C D$ rates have been report$\mathrm{ed}^{7}$, but the findings are not yet recommended for practice in many maternity units. Notably, a recent study (n $=42$ 539) showed that prolonged duration of the second stage of labour is associated with adverse neonatal outcomes ${ }^{3}$. Despite the lack of robust evidence to guide the acceptable duration of labour when there is fetal demise, the authors recommend that the same timelines used for the labour of a live fetus may be applied. In authors' opinion, if there is fetal demise in a primigravida in a maternity unit that provides one patient to one midwife monitoring, the duration of second stage of labour may be arguably marginally exceeded (preferably a total duration not more than 4 hours) after confirming progressive descent of the presenting part, satisfactory maternal condition, and obtaining informed consent from the patient. This marginal extension may be acceptable because the second stage of labour with normal outcomes in a primigravida may occasionally last for approximately 4 hours particularly with the use of epidural analgesia ${ }^{8}$. (d) Sequential use of vacuum and forceps for assisted vaginal delivery is associated with maternal ${ }^{9}$ and neonatal injuries ${ }^{10}$ and should not be a routine practice. Medical practitioners should always document the criteria for operative vaginal delivery, and before the procedure indicate if a patient satisfies these conditions. (e) Maternal pre-operative haemoglobin concentration should be performed before a surgical procedure such as CD. Therefore, a ward haemoglobin measuring machine should be available for use in all maternity units. A low haemoglobin may assist with the diagnosis of an occult uterine rupture. (d) Each maternity unit should have alternative emergency means of obtaining advice when the primary telephone lines are faulty. The failure to obtain timely advice possibly could have been prevented by using mobile phones or by sending an emissary to the senior doctor's residence. Highlights are shown in Table 1.

Table 1. Highlights

Induction of labour (IOL) at an inappropriate healthcare facility is unsafe.

Extended duration of labour, even with fetal demise, particularly in a multiparous woman is hazardous.

There is limited data on the duration of labour in women with fetal demise.

Timelines for monitoring during IOL and labour with a demised fetus are proposed.

\section{Conclusion}

Induction of labour induction should be conducted in a healthcare facility with the capacity to safely perform the procedure. Further studies are required to determine the duration of labour when there is fetal demise because fetal health status may influence the event. However, the timelines proposed by the authors are pragmatic and reasonable. Fetal demise should not becloud adherence to monitoring and timely interventions during labour. Unsafe labour management begets gruesome outcomes.

\section{Conflicts of interest}

Authors declared they have no conflicts of interest.

\section{Funding}

The authors did not receive any funding to report this case report.

\section{References}

1. National Collaborating Centre for Women's and Children's Health (UK), Commissioned by the National Institute for Health and Clinical Excellence. Induction of labour. London: RCOG Press; 2008.

2. Society of Obstetricians and Gynaecologists of Canada (SOGC). Induction of Labour: Review. J Obstet Gynaecol Can. 2015;37(4):380-1.

3. Sandström A, Altman M, Cnattingius S, Johansson S, Ahlberg M, Stephansson O. Durations of second stage of labor and pushing, and adverse neonatal outcomes: a population-based cohort study. J Perinatol. 2017;37(3):236-42. 
4. National Committee on the Confidential Enquiries into Maternal Deaths. Saving Mothers 2014-2016: Seventh triennial report on confidential enquiries into maternal deaths in South Africa: Short report. Pretoria: South African Department of Health 2018.

5. South African National Department of Health. Standard treatment guidelines and essential medicines list for South Africa: Hospital level adults. 2015; Available from: https://www.samedical.org/cms_uploader/ viewFile/447 (accessed 26 May 2019).

6. World Health Organization. WHO recommendations: intrapartum care for a positive childbirth experience. Geneva: World Health Organization; 2018. Licence: CC BY-NC-SA 3.0 IGO. Available from: https:// www.who.int/reproductivehealth/publications/intrapartum-care-guidelines/en/ (accessed 26 May 2019).
7. Gimovsky AC, Berghella V. Randomized controlled trial of prolonged second stage: extending the time limit vs usual guidelines. Am J Obstet Gynecol. 2016;214(3):361. e1-6.

8. Abalos E, Oladapo OT, Chamillard M, Díaz V, Pasquale J, Bonet M, et al. Duration of spontaneous labour in 'low-risk' women with 'normal' perinatal outcomes: A systematic review. Eur J Obstet Gynecol Reprod Biol. 2018;223:123-32.

9. Society of Obstetricians and Gynaecologists of Canada (SOGC). No. 381-Assisted Vaginal Birth. J Obstet Gynaecol Can. 2019;41(6):870-82.

10. Gardella C, Taylor M, Benedetti T, Hitti J, Critchlow C. The effect of sequential use of vacuum and forceps for assisted vaginal delivery on neonatal and maternal outcomes. Am J Obstet Gynecol. 2001;185(4):896-902. 